\title{
An experimental method to investigate the role of low frequency phenomena in the generation of tidal residues*
}

\author{
MIGUEL BRUNO, RAFAEL MAÑANES, JOSÉ JUAN ALONSO, ALFREDO IZQUIERDO \\ and OSCAR ALVAREZ
}

Faculty of Marine Sciences. Polígono del Río San Pedro s/n. Puerto Real Aptdo. 40. Cádiz. Spain.

\begin{abstract}
SUMMARY: In this work a method to estimate the contribution to tidal residues originated by non-linear interaction between tides and low frequency phenomena is presented. In order to perform the validation, the method is applied on current data series taken from the Strait of Gibraltar which allows us to find a clear relationship between current tidal residues and low frequency fluctuations in the flow, which may be explained in terms of the non-linear interaction between tidal and sub-inertial signals in the current velocity.
\end{abstract}

Key words: tidal hydrodynamics, shallow water tides, non-linear interactions.

\section{INTRODUCTION}

In the context of this work, tidal residues are defined as the signals which remain in tidal bands after removing the predicted astronomical tidal signal from the original recording. The remaining residual signal is basically due to the slow time variations in the harmonic constant values, amplitude and phase lag, of the main tidal constituents. Amplitude and phase variations may be due to modulations by any non-included minority constituents in the prediction (Amin, 1976) or modulations from a non-astronomical origin.

Many authors have related a large part of these non-astronomical residues to the non-linear interaction between tidal signal and low frequency phe-

\footnotetext{
*Received January 20, 1998. Accepted May 7, 1999.
}

nomena. Indeed, Munk et al. (1964) proposed this mechanism to explain the modulations presented by several harmonic constituents in sea level records. Rossiter and Lennon (1968) proposed the astronomical tide - low frequency surge interaction to explain the semi-diurnal residues arising from sea level records. García (1986) used the Munk et al. mechanism to explain a large part of the semi-diurnal residue variance of several sea level recordings taken in the Strait of Gibraltar. Bruno et al. (1996) explain the semi-diurnal residue of sea level recording taken at Gran Canaria Island by characterizing this non-linear interaction between sub-inertial and tidal signal by means of an analytical solution. Using a similar procedure as the last authors, Mañanes (1996) and Mañanes et al. (1997) explain the semi-diurnal residue in the current velocity along the Strait of Gibraltar. 
In the present work, a method to estimate the contribution of these non-linear interactions to tidal residues in sea level and current velocity data recordings is presented. A formulation to characterize tidal residues as a function of slow time variations in amplitude and phase of the main tidal constituents is developed in Section one. In Section two the applied procedure on data recording to extract the tidal residues from them in accordance with the proposed formulation. In Section three the procedure is applied on current data series taken from the Strait of Gibraltar in the investigation of the relationship between tidal residues and the sub-inertial signal in the current velocity. Finally a physical reading of the tidal current residues in terms of the non-linear interaction between tidal and sub-inertial flows is presented in Section four.

\section{TIDAL RESIDUES AS A FUNCTION OF SLOW TIME AMPLITUDE AND PHASE VARIATION OF THE MAIN TIDAL CONSTITUENTS}

The signal of any tidal constituent affected by some non-linear interaction between tide and some low frequency phenomena can be expressed (Bruno et al., 1996) as

$$
s_{T}^{d}(t)=A_{m}(t) \cos \left[\omega_{T} t-\phi_{m}(t)\right]
$$

where $S^{d}{ }_{T}(t)$ is the distorted signal of the tidal constituent $T$, and $A_{m}(t)$ and $\phi_{m}(t)$ are its slow amplitude and phase lag variations in time.

On the other hand, the tidal residue associated with such a distortion can then be expressed as

$$
s_{r}(t)=s_{T}^{d}(t)-A_{T} \cos \left(\omega_{T} t-\delta_{T}\right)
$$

where $A_{T}$ and $\delta_{T}$ are the estimated harmonic constants, amplitude and phase, of the $T$ signal and $\omega_{T}$ its angular frequency. The term $s_{T}^{d}(t)$ in Eq. (2) can be expressed in the alternative way

$$
s_{T}^{d}(t)=A_{T} A_{t} \cos \left(\omega_{T} t-\delta_{T} \phi_{t}\right)
$$

where

$$
A_{t}=\frac{A_{m}(t)}{A_{T}}
$$

and

$$
\phi_{t}=\frac{\phi_{m}(t)}{\delta_{T}}
$$

are non-dimensional factors which express the proportion of amplitude and phase variations with respect to the harmonic constants for the $T$ tidal signal. Developing Eq. (3) as a function of $A_{t}$ and $\phi_{t}$ by Taylor's series around the undistorted signal, $A_{t}=1$ and $\phi_{t}=1$, and truncating it in the first order results in

$$
s_{T}^{d}(t) \approx s_{T}^{d}(1,1)+\left.\frac{\partial s_{T}^{d}}{\partial A_{t}}\right|_{(1,1)}\left(A_{t}-1\right)+\left.\frac{\partial s_{T}^{d}}{\partial \phi_{t}}\right|_{(1,1)}\left(\phi_{t}-1\right) .
$$

Evaluating the partial derivatives in $A_{t}=1$ and $\phi_{t}=1$, and taking in to account that

$$
s_{T}^{d}(1,1)=A_{T} \cos \left(\omega_{T} t-\delta_{T}\right)
$$

the following equation is obtained

$$
\begin{gathered}
s_{r}(t)=s_{T}^{d}(t)-A_{T} \cos \left(\omega_{T} t-\delta_{T}\right) \approx \\
A_{T} \cos \left(\omega_{T} t-\delta_{T}\right)\left(A_{t}-1\right)+\delta_{T} A_{T} \sin \left(\omega_{T} t-\delta_{T}\right)\left(\phi_{t}-1\right) .
\end{gathered}
$$

Therefore, the right hand side of expression (5) can be considered as an approximation to the tidal residue $s_{r}(t)$ given by Eq. (2). From Eq. (5), the residual variance can be divided into two parts. The first part is proportional to the $T$ signal

$$
s_{r_{1}}=C_{l} \cos \left(\omega_{T} t-\delta_{T}\right)
$$

where

$$
C_{l}=A_{T}\left(A_{t}-1\right)
$$

explaining the contribution to residue by the amplitude variations of the $T$ signal. The second part is proportional to an orthogonal phase lag signal with respect to the $T$ one

$$
s_{r_{2}}=S_{l} \sin \left(\omega_{T} t-\delta_{T}\right)
$$

where

$$
S_{l}=\delta_{T} A_{T}\left(\phi_{t}-1\right)
$$

explaining the contribution to residue by the phase lag variations of the $T$ signal. The different contributions to tidal residue from amplitude and phase lag variations on the $T$ signal can thus be evaluated.

The tidal residues can now be characterized by the low frequency series: $C_{l}$ and $S_{l}$, which represent the modulated amplitudes of the tidal residues, and could be used to study any relationship of the later with some low frequency variable later. Also, the values $C_{l}$ and $S_{l}$ yield a direct way to compare the different contributions to tidal residues from amplitude and phase variation of the main tidal constituent, which cannot be clearly 
seen using the $A_{m}$ and $\phi_{m}$ series presented in Eq. (1) directly.

The procedure described above can only be directly applied to a hypothetical record consisting of only one distorted tidal constituent. However, in the observed recording the tidal residues are generated by the amplitude and phase variation of some more constituents and the present approach to tidal residue cannot be applied in such a direct way.

The characterisation of the tidal residues using the approach given by Eq. (5) can be performed, assuming that inside each tidal band the distortion on the main tidal constituent (i.e. $\mathrm{M}_{2}$ and $\mathrm{K}_{1}$ for semi-diurnal and diurnal tidal bands respectively) represents the distortion felt by the whole of the tidal band. This is in accordance with the credo of smoothness.

To isolate the distorted signal of a main constituent of any tidal band in data recording, any contributions from other secondary constituents inside the same band must be eliminated to avoid modulations of the latter on the main constituent. Once this is done, the resulting signal will represent the distorted tidal signal which can be expressed using Eqs. (1) and (3); the residual signal associated with this distortion can then be expressed through Eqs. (2) or (5).

\section{THE APPLICATION OF THE METHOD ON OBSERVED TIDAL RECORDS}

To characterise the tidal residues arising from tidal recording in accordance with the formulation developed in the previous section, certain details about the preprocessing of the original recording must be considered. It is neccesary to distinguish the preprocessing concerning the sea level records from that concerning the current velocity recording. This is because to characterize the sea level signal in some location, inside a given oceanic region, only one recording can be enough while the characterization of the current velocity usually demands several recordings at different depths throughout the water column. However, many times it is necessary to reduce the current velocity signal recorded at different depths onto only one signal which is representative of the behaviour of the whole of the water column portion where the measurements have been taken. This reduction is, for example, always required when the comparison between the observed current velocity data and those provided by depth averaged analytical or numerical models.

In the case of a homogeneous water column and therefore, a basically barotropic behaviour of flow, the current velocity recording must show similar behaviour except in the vicinity of the sea surface and bottom. In this way, a simple vertical average of the measurements provide a representative for the whole of the water column current velocity.

In the case of a stratified water column, it can be helpful to us the two-layer approach, the depth averaging for each layer of current velocity measurements will provide a representative current velocity for each one.

Amplitude and phase lag variation, $A_{m}(t)$ and $\phi_{m}(t)$, of a main tidal signal from sea elevation or current velocity recordings may be obtained as follows:

1. Isolate the desired tidal band by application of a band pass Fast Fourier Transform (FFT) filter or any equivalent filtering procedure on the original signal. In the case of velocity measurements at different depths, it is necessary to reduce the velocity measurements to a suitable average with depth.

2. Apply the least squares harmonic analysis on the isolated signal to get the estimation of the harmonic constants for the main tidal constituents of the band.

3. From the above results generate and eliminate from the signal a prediction without the main tidal constituent. The resulting signal is thus composed of the signal of the main constituent plus the tidal residue and can be understood as a main constituent distorted signal which can be characterized by the Eq. (1).

4. Obtain amplitude and phase variation of the $T$ signal, $A_{m}(t)$ and $\phi_{m}(t)$, by application of complex demodulation (Garrett et al., 1989; Bruno et al., 1996; Mañanes, 1996) at the $T$ frequency on distorted $T$ signal series.

With $A_{t}=A_{m}(t) / A_{T}$ and $\phi_{t}=\phi_{m}(t) / \delta_{T}$ obtained from the last step of the procedure above, then the low frequency series, $C_{l}$ and $S_{l}$, which characterize the modulated amplitudes of the tidal residues can be evaluated. If tidal residue were related to some low frequency variable, $C_{l}$ and $S_{l}$ could be used to study the possible relationships between them and the given low frequency variable, making possible the identification of non-linear phenomena affecting the tidal signal in real data record. 


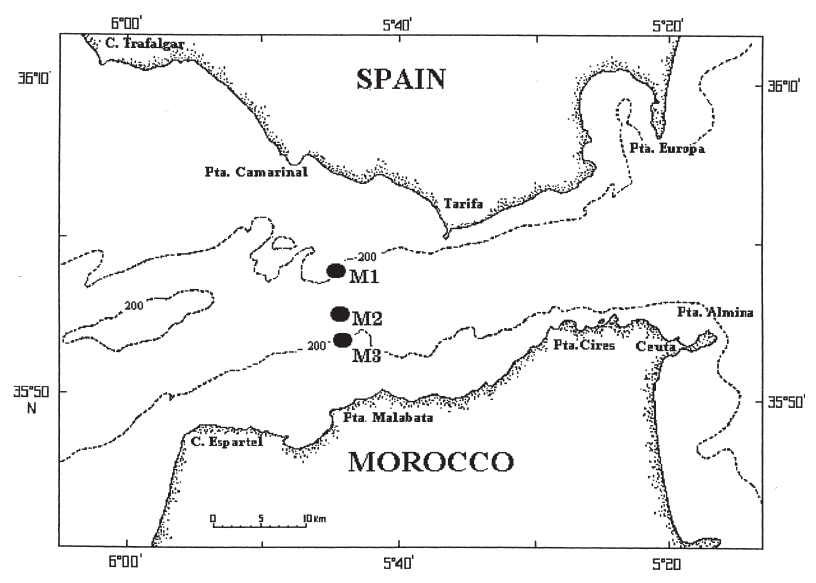

FIG. 1. - Map of the studied zone, with the location of the moorings deployed in the Gibraltar experiment (1985-1986). Depth is expressed in meters.

\section{A PRACTICAL APPLICATION CASE ON CURRENT VELOCITY RECORDS}

To show an example of the above procedure in the investigation of the non-linear interaction phenomenon between tidal and low frequency (subinertial) flows, some results on marine current data record taken in the Strait of Gibraltar are given. The data derive from the Gibraltar Experiment (1985-1986) and they were taken from moorings M1, M2 and M3, located in the Camarinal Sill cross-section (Fig. 1). The maximum length of the simultaneous recording period was about one month. Mooring M3, where a simultaneous three months hourly record had been achieved, was selected for further analysis. The measurements of the other moorings are helpful for the physical interpretation of the results. M3 mooring was composed of three currentmeters located at 110 , 140 and 180 meters depth (see Table 1). The semi- diurnal tidal band was selected for analysis because it explains more than $70 \%$ of the tidal current velocity variance in the Strait of Gibraltar (Candela et al., 1990). Due to the basically onedirectional character of current velocity in the Strait a clear predominance of the West-East component of the current velocity $u$ on the SouthNorth component $v$ exists (Pillbsbury et al., 1987). The current velocity in the later analysis will be characterized by the $u$ component.

It should be noted that as the mean position of the interface separating the Atlantic and Mediterranean waters is about 100 meters depth in this zone, all the currentmeters of the three moorings (Table 1) were located in the lower layer. Therefore, the current velocity measurements are only representative of the deeper layer.

\section{Characterisation of the semi-diurnal residue in the current velocity}

The characterisation of the barotropic semi-diurnal residue starts with the application of the preprocessing sequence proposed in the previous Section, on the original $u$ component series recorded by each one of the M3 currentmeters.

1. Isolation of the semi-diurnal tidal band by a band pass FFT filter on the original current velocity series. The cut-off frequencies for the semi-diurnal band are 0.07-0.09 cycles/hour. Instantaneous semidiurnal signal time series are then averaged with depth to obtain a representative for the lower layer semi-diurnal signal. Hereinafter, the latter signal is designed as 'averaged semi-diurnal signal'.

2. A least squares harmonic analysis (Foreman, 1976) was applied on the averaged semi-diurnal signal to estimate the harmonic constants for the main semi-diurnal tidal constituents.

TABLE 1. - Some information about the moorings deployed in the Gibraltar Experiment (1985-1986).

\begin{tabular}{|c|c|c|c|c|c|c|}
\hline Mooring & Water Depth (m) & Latitude & Longitude & Start & Stop & Depth of Instrument (m) \\
\hline M1 & $222 \mathrm{~m}$ & $35^{\circ} 58.26^{\prime}$ & $05^{\circ} 44.62^{\prime}$ & $\begin{array}{l}22 / 10 / 85 \\
22 / 10 / 85 \\
22 / 10 / 85 \\
22 / 10 / 85\end{array}$ & $\begin{array}{l}04 / 05 / 86 \\
04 / 05 / 86 \\
04 / 05 / 86 \\
04 / 05 / 86\end{array}$ & $\begin{array}{l}143 \mathrm{~m} \\
156 \mathrm{~m} \\
167 \mathrm{~m} \\
215 \mathrm{~m}\end{array}$ \\
\hline M2 & $310 \mathrm{~m}$ & $35^{\circ} 54.74^{\prime}$ & $05^{\circ} 44.55^{\prime}$ & $\begin{array}{l}22 / 10 / 85 \\
22 / 10 / 85 \\
22 / 10 / 85 \\
22 / 10 / 85 \\
22 / 10 / 85\end{array}$ & $\begin{array}{l}23 / 11 / 85 \\
23 / 11 / 85 \\
23 / 11 / 85 \\
23 / 11 / 85 \\
23 / 11 / 85\end{array}$ & $\begin{array}{l}123 \mathrm{~m} \\
143 \mathrm{~m} \\
153 \mathrm{~m} \\
254 \mathrm{~m} \\
306 \mathrm{~m}\end{array}$ \\
\hline M3 & $190 \mathrm{~m}$ & $35^{\circ} 53.42^{\prime}$ & $05^{\circ} 44.20^{\prime}$ & $\begin{array}{l}21 / 10 / 85 \\
21 / 10 / 85 \\
21 / 10 / 85\end{array}$ & $\begin{array}{l}21 / 4 / 86 \\
21 / 4 / 86 \\
21 / 4 / 86\end{array}$ & $\begin{array}{l}110 \mathrm{~m} \\
140 \mathrm{~m} \\
180 \mathrm{~m}\end{array}$ \\
\hline
\end{tabular}


a)

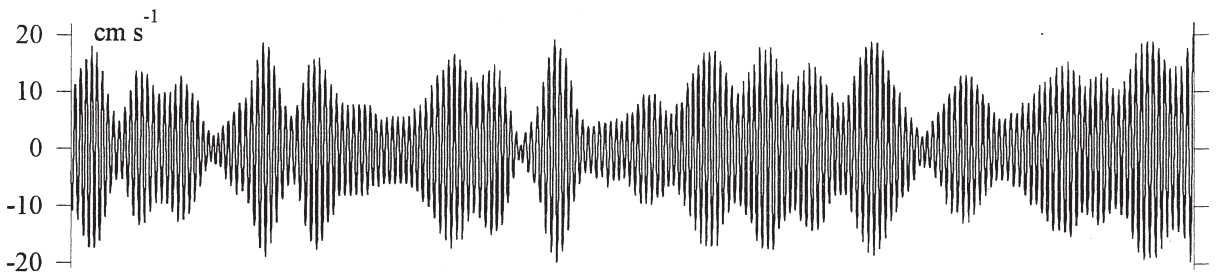

b)
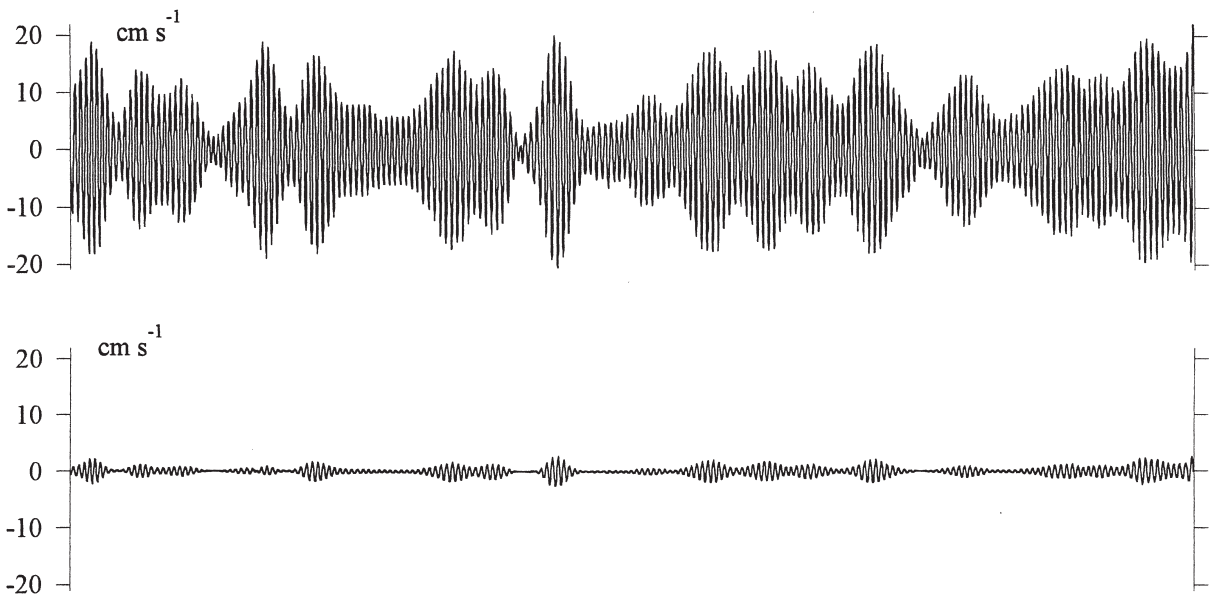

c)

d)
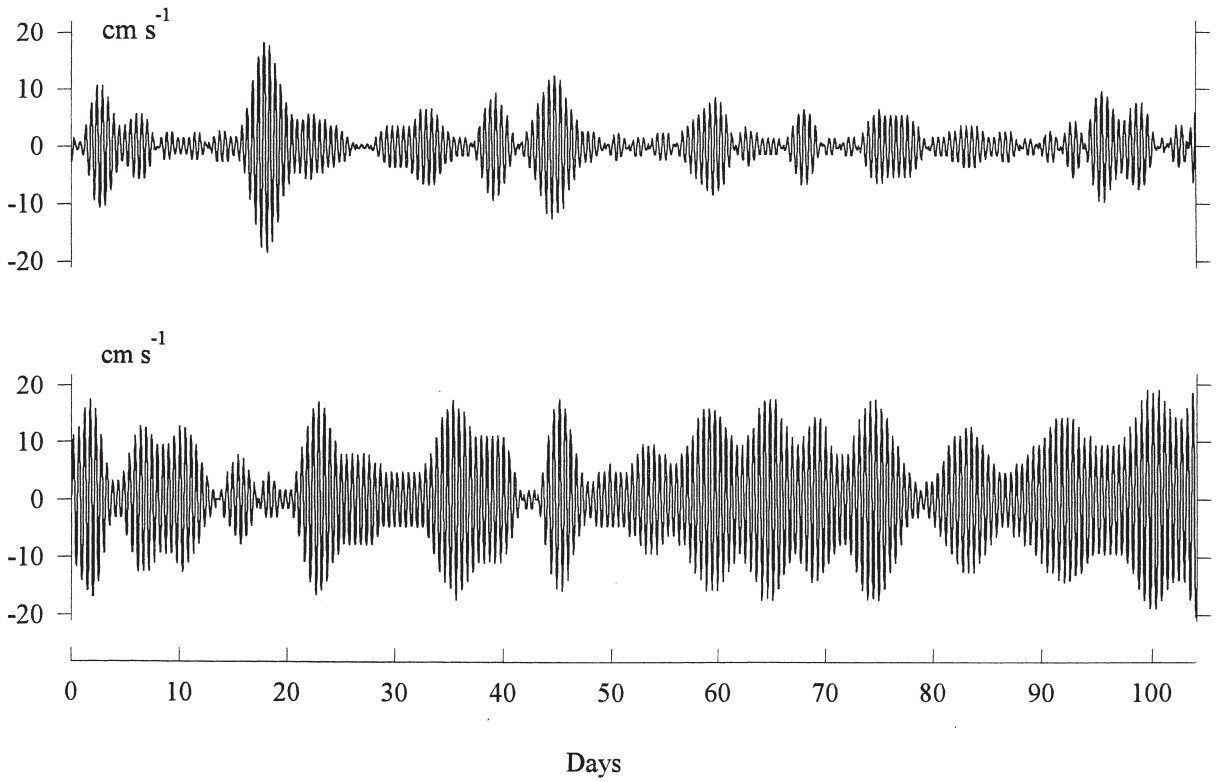

FIG. 2. - Time series of the semi-diurnal residue in the current velocity at mooring M3. a) observed residue $s_{r}(t)$; b) approximated residue $s_{r a}(t)$; c) difference between the observed and approximated residues; d) contribution to the semi-diurnal residue from $\mathrm{M}_{2}$ signal amplitude variation $s_{r 1}(t)$; and e) contribution to the semi-diurnal residue from the $\mathrm{M}_{2}$ signal phase variation $s_{r 2}(t)^{2}$.

3. A prediction without $\mathrm{M}_{2}$ constituent is generated using the previously estimated harmonic constants and it is subtracted from the averaged semidiurnal signal. The resulting signal is composed of the $\mathrm{M}_{2}$ signal plus the semi-diurnal residue and can be understood as a distorted $\mathrm{M}_{2}$ signal which can be characterized in the manner of Eq. (1).
4. The complex demodulation is applied on the distorted $\mathrm{M}_{2}$ signal to obtain the slow time amplitude and phase variations series of the $\mathrm{M}_{2}$ signal, $\mathrm{A}_{\mathrm{m}}$ and $\phi_{\mathrm{m}}$. Next the low frequency terms $\mathrm{C}_{1}$ and $\mathrm{S}_{1}$, characterising the semi-diurnal tidal residue from amplitude and phase variations of the $\mathrm{M}_{2}$ signal, are obtained. 
TABLE 2. - Statistics of semi-diurnal residues at M3 mooring; mean, standard deviation, minimum and maximum values. s means for the observed residue; $s_{n}$ for the approximated residue; $s_{r 1}$ for the contribution to the semi-diurnal residue from the $M_{2}$ signal amplitude variation; $\mathrm{s}_{\mathrm{r} 2}$ for the contribution to the semi-diurnal residue from the $\mathrm{M}_{2}$ signal phase variation; and $\mathrm{s}_{\mathrm{DIF}}$ for the difference between the observed and approximated residue.

\begin{tabular}{|c|c|c|c|c|c|}
\hline & $S_{r}\left(\mathrm{~m} \mathrm{~s}^{-1}\right)$ & $S_{r a}\left(\mathrm{~m} \mathrm{~s}^{-1}\right)$ & $S_{D i f}\left(\mathrm{~m} \mathrm{~s}^{-1}\right)$ & $S_{r l}\left(\mathrm{~m} \mathrm{~s}^{-1}\right)$ & $S_{r 2}\left(\mathrm{~m} \mathrm{~s}^{-1}\right)$ \\
\hline$\sigma$ & 0.083 & 0.084 & 0.0070 & 0.036 & 0.076 \\
\hline Max. & 0.229 & 0.220 & 0.027 & 0.181 & 0.190 \\
\hline Min. & -0.197 & -0.204 & -0.025 & -0.184 & -0.202 \\
\hline
\end{tabular}

Figure 2 shows the residual series estimated from Eq. (2), the 'observed residue' and its approximation from Eq. (5), the 'approximated residue', during the analyzed period at mooring M3. Also, the difference between observed and approximated residues is shown. In Table 2, the statistics of the residuals are presented. It should be noted that the quotient between the standard deviations of the series in Figure 2.c and the series in Figure 2.a is 0.084 and therefore the approximation to the semidiurnal residue by Eq. (5) is quite satisfactory.

The different contributions to semi-diurnal residue from amplitude and phase variations of $\mathrm{M}_{2}$ signal computed by Eq.(6) and (7) respectively (see Figs. 2.d and 2.e). In Table 2, the standard deviations of those contributions are presented. It must be noted that the contribution to semi-diurnal residue of phase variation is near twice that of amplitude variation.
Once the series $C_{l}$ and $S_{l}$ are obtained (see Fig. 3), their possible relationships with the low frequency part of the current velocity oscillation, the sub-inertial oscillation of the current velocity, can be investigated.

\section{Attainment of the sub-inertial oscillation of the current velocity}

The sub-inertial oscillation of the current velocity was obtained after application of the moving average filter $\mathrm{A}_{24} \mathrm{~A}_{24} \mathrm{~A}_{25}$ (Godin, 1972), which has a cut-off frequency of 0.033 cycles/hour corresponding to a period of 30 hours, on the original $u$ component series to yield the sub-inertial signal at all the currentmeters of M3 mooring. Thereafter, the depth average of the instantaneous sub-inertial signal yielded a representative for the lower layer sub-inertial velocity time series. Hereinafter this signal will be named $u_{l}$ (see Fig. 3).
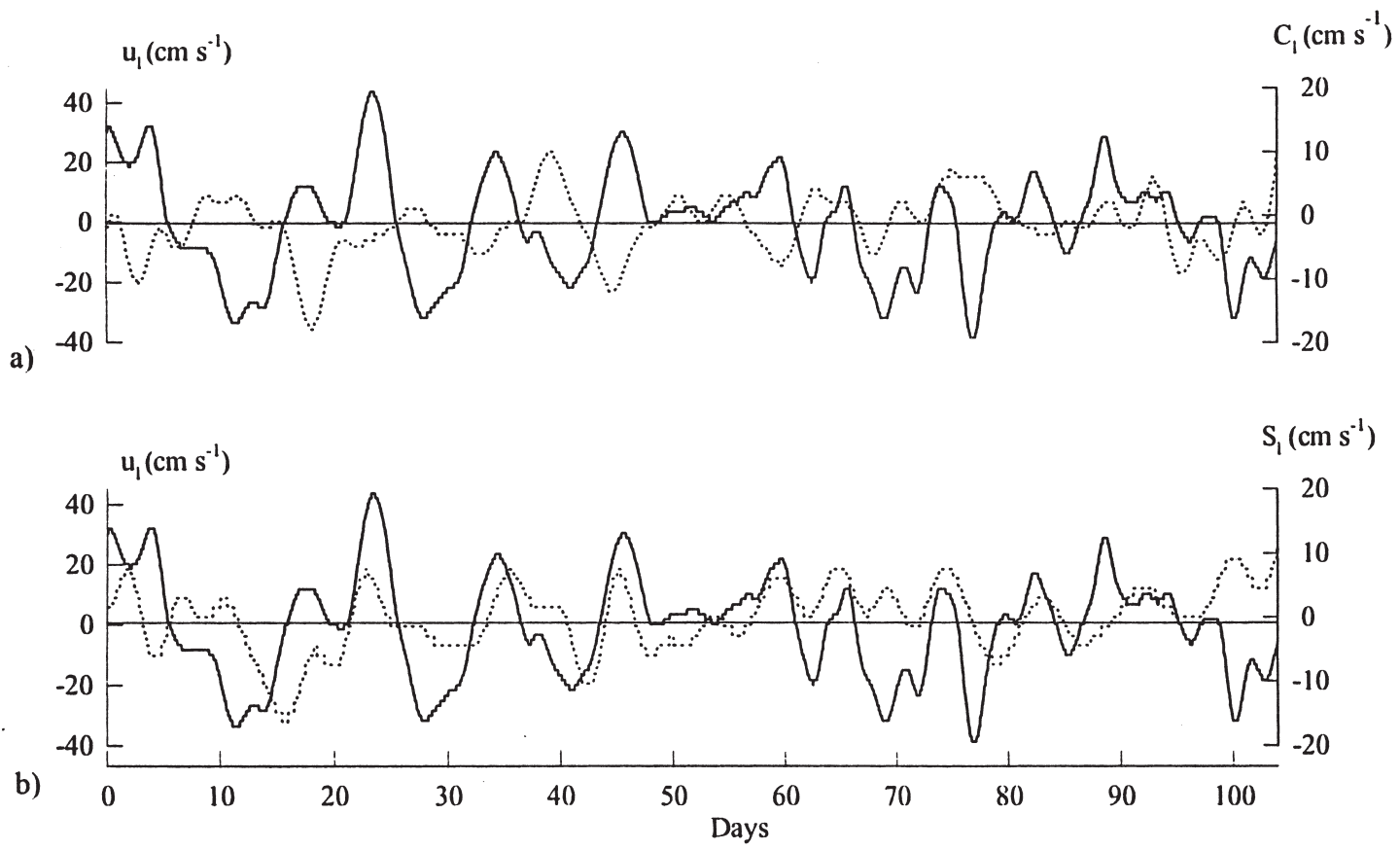

FIG. 3. - Low frequency terms, $C_{l}(t)$ and $S_{l}(t)$, arising from the semi-diurnal tidal current residue characterisation through Eq. (5) together with depth averaged sub-inertial oscillation in the current velocity $u_{l}(t)$ in the mooring M3. a) $C_{l}(t)$ (dashed line) and $u_{l}(t)$ (solid line), and b) $S_{l}(t)$ (dashed line) and $u_{l}(t)$ (solid line). 

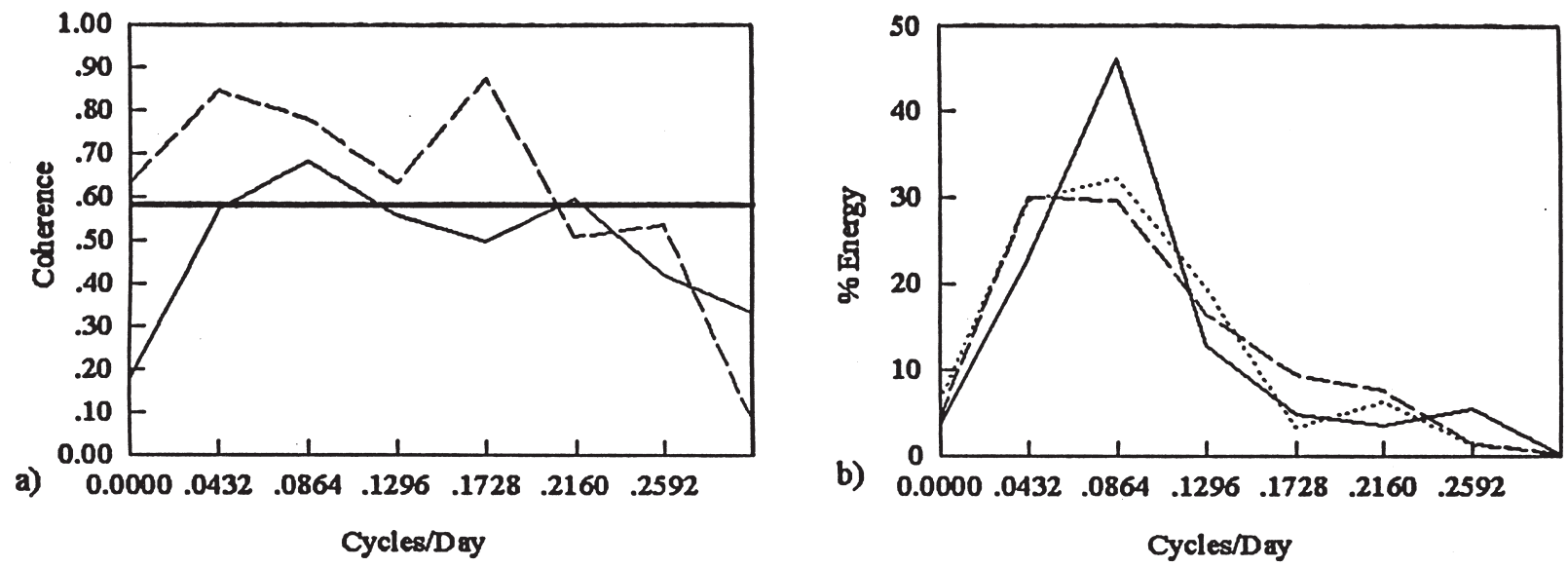

FIG. 4. - Results of the cross spectral analysis between $C_{l}(t), S_{l}(t)$ series and $u_{l}(t)$ series at the mooring M3. a) coherence diagram; $C_{l}(t)$ vs $u_{l}(t)$ (solid line) and $S_{l}(t)$ vs $u_{l}(t)$ (dashed line), horizontal line means for 95\% significant level for coherence in accordance with Box and Jenkins $(1970)$; b) percentage of variance explained for each frequency band - $C_{l}(t)$ (solid line), $S_{l}(t)$ (dashed line) and $u_{l}(t)($ dotted line). Spectral estimations have been made with 9 degrees of freedom and a band width of $\Delta \omega=0.0432$ cycles/day.

\section{Relationship of $C_{l}$ and $S_{l}$ with $u_{l}$}

In Figure 3 certain relationships between both pairs of series $u_{l}(t)$ with $C_{l}(t)$ and $S_{l}(t)$ for mooring M3 can be observed from visual inspection. To look into these relationships, a cross spectral analysis between both pairs of terms was performed. Results from this analysis are shown in Figure 4. In the frequency range where the explained percentage of energy is higher for all terms (from 0.0432 to 0.1296 cycles/day) (Fig. 4.b), the coherence shows significant values, between 0.5 and 0.8 , implying a significant correlation of the two pairs of terms. At this state of the analysis exists a significant correlation between sub-inertial oscillation and the semi-diurnal residue in the current velocity.

It is very interesting that the coherence values presented by the $S_{l}$ terms are greater than those of $C_{l}$. Also the $S_{l}$ term presents a strong similarity with $u_{l}$ as the percentage of explained variance distribution concerns (Fig. 4.b). This means that the phase variation contributes to semi-diurnal residue nearly twice that of amplitude variation (Table 2). Thus, the major part of the semi-diurnal residue variance is highly correlated with sub-inertial oscillation in the flow.

The above analysis has been made possible using the procedure proposed in the previous Section to express semi-diurnal tidal current residues in accordance to the formulation developed in the first Section. In the next section the existing correlation will be interpreted as a result of the non-linear interaction between semi-diurnal and sub-inertial signals in the current velocity.

\section{PHYSICAL ORIGIN OF SEMI-DIURNAL RESIDUES IN THE CURRENT VELOCITY}

From results of the previous section, the existence of a significant relationship between $\mathrm{M}_{2}$ signal amplitude and phase distortion with respect to subinertial oscillation in the current in the frequency range from 0.0432 to 0.1296 cycles/day can be affirmed. It is in this range where both distortion and sub-inertial oscillation present most of their variances and where the coherence is higher.

As commented in the introduction, a physical mechanism that could explain the generation of the semi-diurnal residues related to the sub-inertial oscillation in the current is the non-linear interaction between tidal and sub-inertial flows.

An analytical explanation of this phenomenon can be given if one considers the Straits hydrodynamics to be characterized by a two-layer system, where inside each layer flow exist only in the alongstrait direction. Then, the vertically integrated Equations of motion along each layer may be written as

$$
\frac{\partial u}{\partial t}+u \frac{\partial u}{\partial x}=-\frac{1}{\rho} \frac{\partial P}{\partial x}+\frac{\tau_{\zeta}-\tau_{d}}{h+\zeta}
$$

and

$$
f u=-\frac{1}{\rho} \frac{\partial P}{\partial y}
$$

where in the integration, the vertical co-ordinate has been considered as positive downward; $x$ and $y$ are the along-strait and cross-strait co-ordinates; $u$ is the along-strait velocity of the flow; $\rho$ is the sea-water density of the layer; $P$ is the hydrostatic pressure; $h$ is the time averaged thickness of the layer; $\zeta$ is the 
vertical distance over its mean position for the sea surface, in the upper layer, or for the interface, in the lower layer; $f$ is the Coriolis parameter; and, $\tau_{\zeta}$ and $\tau_{d}$ represent, respectively, the shear stress in the upper and lower surfaces bounding each layer.

The balance of forces indicated by Eqs. (8) and (9) can be a reasonable approximation to the dynamic of the flow through the Strait, at least, in the Camarinal Sill cross-section where a denser arrangement of current velocity measurements has been developed in historical surveys. Several works such as those by Pillsbury et al. (1987) and Candela et al. (1990) have reported that in the Camarinal Sill the flow is practically aligned with the longitudinal axis of the Strait. Also, following Candela et al. (1990) and Bray et al. (1991), the flow is highly correlated with the cross-strait component of the horizontal pressure gradient and it seems that, in a first order approach the cross-strait component of the Coriolis force is balanced by the cross-strait component of the horizontal pressure gradient force. Finally, the results of a high-resolution boundary fitted tidal model of the Strait (Tejedor et al., 1998) support this approach for the Camarinal Sill, at least for the main semi-diurnal tidal waves dynamic.

Our purpose now will be to estimate the relative magnitude order of the non-linear terms presented in Eq. (8) with respect to the linear signal, i. e. the local acceleration term. With this exercise we will obtain analytically based quantities which may be asimilated as quotients between the amplitude of the nonlinearly generated semi-diurnal residue and the amplitude of the $\mathrm{M}_{2}$ linear signal. In order to do this, one-month long simultaneous current velocity recording from moorings M1 and M2 located along the Camarinal Sill cross-section (see Fig. 1) are used. It must be noted that all the current meters were below the mean interface position and therefore the velocity measurements are only representatives of the lower layer at the Camarinal Sill crosssection. In this way, further computations will be based on the use the Eqs. (8) and (9) on the lower layer.

Before computing these magnitude orders, it is necessary to explore some ideas about the friction term approach presented in Eq. (8). In the lower layer the shear stress in the interface is neglected against the bottom stress and the latter will be expressed as

$$
\tau_{d}=k|u| u
$$

where $k$ is the bottom stress coefficient. Eq. (8) for the lower layer thus becomes

$$
\frac{\partial u}{\partial t}+u \frac{\partial u}{\partial x}=-\frac{1}{\rho} \frac{\partial P}{\partial x}-\frac{k|u| u}{h+\zeta} .
$$

Neglecting the shear stress at the interface may be justified because of the strong stratification of the water column existing in the Strait of Gibraltar which prevents the vertical momentum exchange through the interface. In this way, the eddy viscosity coefficient in the vicinity of the interface takes small values originating relatively small values of shear stress. Of course, this asumption fails during the ocurrence of large internal bores generation and other short period internal waves existing in the Strait, which increase the turbulence in the interface and therefore the interfacial friction. This may take place along the hydraulic jump formed west of Camarinal sill during the ebb tide (Armi and Farmer, 1988). Nevertheless, considering the temporal and spatial scales associated with the tidal dynamics in the Strait, the spatial and temporal variations of interfacial shear stress associated with the mentioned causes suppose only local (in both, space and time) perturbations over the mean field of shear stress.

Next the variables $\zeta$ and $u$ are considered as compound of

$$
\zeta=\zeta_{M_{2}}+\zeta_{l}
$$

and

$$
u=u_{M_{2}}+u_{l}
$$

denoting that the linear solution consist of the $\mathrm{M}_{2}$ signal; $\zeta_{M 2}$ and $u_{M 2}$; plus a sub-inertial signal; $\zeta_{l}$ and $u_{l}$.

Using the approach of Godin and Martínez (1994) for bottom friction

$$
-\frac{k|u| u}{h+\zeta} \approx-\frac{k m}{2 h} u+\frac{m k}{2 h^{2}} u \zeta-\frac{k}{2 h m} u^{3}+\frac{k}{2 h^{2} m} \zeta u^{3}
$$

where $\mathrm{m}=0.7 ; \mathrm{k}=2.510^{-3}$ and by which the bottom friction term is decomposed in a linear plus three non-linear terms, substituting it into the non-linear terms of Eq. (12) and retaining only the first and second order terms

$$
\begin{gathered}
\frac{\partial u}{\partial t}+u_{l} \frac{\partial u_{M_{2}}}{\partial x}+u_{M_{2}} \frac{\partial u_{l}}{\partial x}+u_{M_{2}} \frac{\partial u_{M_{2}}}{\partial x}+u_{l} \frac{\partial u_{l}}{\partial x}= \\
=-\frac{1}{\rho} \frac{\partial P}{\partial x}-\frac{k m}{2 h} u+\frac{m k}{2 h^{2}}\left(u_{l} \xi_{M_{2}}+u_{M_{2}} \xi_{l}\right) .
\end{gathered}
$$

In order to establish the frequencies where the variance of the non-linear terms presented in this 
Equation are located, the $\mathrm{M}_{2}$ linear signal in the current velocity and interface oscillation will be characterized respectively as

$$
u_{M_{2}}=U_{M_{2}}(x, y) \cos \left[\omega_{M_{2}} t-g_{u_{M 2}}(x, y)\right]
$$

and

$$
\xi_{M_{2}}=A_{M_{2}}(x, y) \cos \left[\omega_{M_{2}} t-g_{\zeta_{M 2}}(x, y)\right]
$$

where $U_{M 2}, A_{M 2}$ are the amplitudes of the oscillation and $g_{u M 2}$ and $g_{\Sigma M 2}$ their phase lags. It is assummed that the sub-inertial velocity oscillation is independent of time during at least a $\mathrm{M}_{2}$ cycle. This assumption will not suppose any fundamental restriction in the further analysis. This means that sub-inertial oscillation behaves in time as a function varying in a step mode where each step has a duration of one $\mathrm{M}_{2}$ cycle. In this way, for each particular $\mathrm{M}_{2}$ cycle, we have a time averaged value of the sub-inertial oscillation which evidently will change step to step. The signal of the $\mathrm{M}_{2}$ acceleration during whatever $\mathrm{M}_{2}$ cycle will receive apart from the linear term (the local acceleration term)

$$
L=\frac{\partial u_{M_{2}}}{\partial t}
$$

additional contributions from the advective acceleration

$$
A=u_{M_{2}} \frac{\partial u_{l}}{\partial x}+u_{l} \frac{\partial u_{M_{2}}}{\partial x}
$$

and bottom friction

$$
B=\frac{m k}{2 h^{2}}\left(u_{l} \zeta_{M_{2}}+u_{M_{2}} \xi_{l}\right)
$$

When the above contributions are translated into velocities they can be understood as semi-diurnal residues in the current velocity since they represent deviations from the pure $M_{2}$ linear signal, which would occur if the sub-inertial oscillation or a mean velocity did not exist.

\section{Relative magnitude orders between non-linear and linear semi-diurnal signals}

The problem now, is evaluating the magnitude order of the linear and non-linear terms presented in the Eqs. (18), (19) and (20) using the available observational data. For this, simultaneous time terms of current velocity recorded at the moorings
M1 and M2 located at the Camarinal Sill cross-section and additional information, such as depth interface observations from the work of Bray et al., (1990) have been used.

As commented in previous Section, the velocity measurements recorded at these moorings were distributed along the lower layer. The observed depth averaged along the lower layer $u_{M 2}$ and $u_{l}$ signals at each mooring were obtained as follows. On the one hand, the $u_{M 2}$ signal was obtained after the least squares harmonic analysis of the recorded time terms at each observational depth, then, the depth averaged amplitude and phase lags were obtained for each mooring. On the other hand, the $u_{l}$ time terms were obtained after applying a $\mathrm{A}_{24} \mathrm{~A}_{24} \mathrm{~A}_{25}$ low pass moving average filter on the time series recorded at each depth, then, an instantaneously depth averaged sub-inertial time series were obtained for each mooring.

The magnitude order of the linear term given by Eq. (18) may be simply evaluated as

$$
[L]=\omega_{M_{2}} U_{M 2} .
$$

The following task is to seek an adequate expression of the spatial derivatives apperaring in Eq. (19) that evaluates the magnitude order of the non-linear terms. For this, Eqs. (8) and (9) particularized to $\mathrm{M}_{2}$ dynamic, at a first order (neglecting non-linear terms), accomplish that

$$
\frac{\partial u_{M_{2}}}{\partial x}=\frac{1}{f} \frac{\partial}{\partial t}\left(\frac{\partial u_{M_{2}}}{\partial y}\right)
$$

the partial derivatives of velocity with respect to the along-strait co-ordinate may be expressed in terms of the derivatives with respect to the cross-strait coordinate.

On the other hand, to evaluate the spatial derivative of the sub-inertial current velocity $u_{l}$ the continuity Equation applied to the lower layer is used

$$
\frac{\partial\left(h u_{l}\right)}{\partial x}=-\frac{\partial \zeta_{l}}{\partial t}
$$

which yields the expression

$$
\frac{\partial u_{l}}{\partial x}=-\left(\frac{u_{l}}{h} \frac{\partial h}{\partial x}+\frac{1}{h} \frac{\partial \zeta_{l}}{\partial t}\right) .
$$

If for the sub-inertial oscillations, the second term on the right hand side of this Equation (taking in consideration the slow time variation of $\xi_{l}$ ) is negligible against the first one, the spatial derivative of $u_{l}$ becomes 


$$
\frac{\partial u_{l}}{\partial x}=-\frac{u_{l}}{h} \frac{\partial h}{\partial x} .
$$

Using Eqs. (22) and (25), the magnitude orders of the non-linear terms given by Eqs. (19) and (20) in a given location of the Strait can then be expressed as

$$
\begin{gathered}
{[A]=U_{M_{2}} \frac{U_{l}}{h} \frac{\partial h}{\partial x}+} \\
+\frac{U_{l} \omega_{M_{2}}}{f} \sqrt{\left(\frac{\partial U_{M_{2}}}{\partial y}\right)^{2}+\left(U_{M_{2}} \frac{\partial g_{u_{M 2}}}{\partial y}\right)^{2}}
\end{gathered}
$$

and

$$
[B]=\frac{m k}{2 h^{2}}\left(U_{l} A_{M_{2}}+U_{M_{2}} A_{l}\right)
$$

where $U_{l}$ and $A_{l}$ represent the characteristic values for the depth averaged velocity and interface sub-inertial oscillations, taken as the standard deviation over the measuring period. Also Eq. (16) has been used in defining the right hand side of Eq. (26).

Using the finite difference approach, these magnitude orders between the moorings M1 and M2 are approximated as follows

$$
\begin{gathered}
{[L]=\omega_{M_{2}} \bar{U}_{M 2}} \\
{[A]=\bar{U}_{M_{2}} \frac{\bar{U}_{l}}{\bar{h}} \frac{\Delta h}{\Delta x}+} \\
+\frac{\bar{U}_{l} \omega_{M_{2}}}{f} \sqrt{\left(\frac{\Delta U_{M_{2}}}{\Delta y}\right)^{2}+\left(\bar{U}_{M_{2}} \frac{\Delta g_{u_{M 2}}}{\Delta y}\right)^{2}}
\end{gathered}
$$

and

$$
[B]=\frac{m k}{2 \bar{h}^{2}}\left(\bar{U}_{l} \bar{A}_{M_{2}}+\bar{U}_{M_{2}} \bar{A}_{l}\right)
$$

where the overbar stands for the averaged value between the two moorings; $\Delta y=8 \mathrm{Km}$ is the acrossstrait distance between the moorings; and $\Delta x=8 \mathrm{Km}$ is the along-strait distance on a segment located at half distance between M1 and M2 and centered on the across-strait segment joining the two moorings.

Now from Eqs. (28) to (30) is defined the quantity

$$
D=\frac{[L]}{[A]+[B]}
$$

that yields a first analytical approximation to the proportion of semi-diurnal residues standard deviation with respect to the $\mathrm{M}_{2}$ linear signal amplitude.

\section{Experimental evaluation of the relative magnitude orders}

The values of the parameters implied in the definition of Eqs. (28)-(30) have been built taking as a basis two different kinds of experimental information. The time series of current velocity recorded at the moorings M1 and M2 (see Fig. 1), on the one hand and the observational data presented in Bray et al. (1990) on the other hand.

Using in Eqs. (28)-(30) the values: $U_{l}=0.2 \mathrm{~m} \mathrm{~s}^{-1}$ deduced from sub-inertial time series obtained at M1 and M2 moorings; $A_{l}=20 \mathrm{~m}, A_{M 2}=40 \mathrm{~m},{ }^{-} \mathrm{h}=$ $220 \mathrm{~m}$ and $\Delta h=120 \mathrm{~m}$, taken from Bray et al. (1990); $U_{M} 0.7 \mathrm{~m} \mathrm{~s}^{-1}$ and $g_{u_{u_{2}}}=164$ for the mooring M1; $U_{M}=0.99 \mathrm{~m} \mathrm{~s}^{-1}$ and $g_{u_{u_{2}}}=150$ for the mooring M2, the computed $D$ value is 0.19 . This value is nearly twice than the proportion of the semi-diurnal residue standard deviation (see Table 2) with respect to the depth averaged $\mathrm{M}_{2}$ velocity amplitude for the mooring M3, $U_{M}=0.9 \mathrm{~m} \mathrm{~s}^{-1}$, which produces a value of 0.092. However, this result supports that the non linear contribution to the acceleration of the flow associated with the $\mathrm{M}_{2}$ dynamics may be an important enough factor to produce the observed semidiurnal residues. It must be noted that the bottom friction term $B$ represents only $2 \%$ of the value of the advective term $A$ meaning that the latter, is the predominant non-linear term in contributing to the semi-diurnal residues variance. Therefore, the nonlinear interaction between $\mathrm{M}_{2}$ and the sub-inertial flows through the advective term may be the physical mechanism to explain the semi-diurnal residues at the Camarinal Sill in the lower layer of the Strait of Gibraltar.

\section{CONCLUSIONS}

In this work, an experimental method to estimate the contribution to tidal residues originated by nonlinear interaction between tides and low frequency phenomena has been presented. The characterisation of tidal residues arising from this methodology yields a direct way to compare the differentiated contribution to residual variance from amplitude and phase variation of the main tidal constituents inside a given tidal band.

An application of the method on current velocity series taken from the Strait of Gibraltar, indicates a clear relationship between sub-inertial fluctuations in the flow and semi-diurnal residues generation. 
For the M3 mooring, the contribution to that residue from the $\mathrm{M}_{2}$ signal phase lag variations is is nearly twice that from amplitude ones. It must be noted that when this kind of non-linear phenomenon is presented, the proposed formulation of tidal residues allows its identification in the time domain, giving the differentiated contribution to semi-diurnal residue from phase and amplitude variation of tidal signals and showing its capability in the study of that kind of phenomenon. Also, the phase variations present higher correlation with sub-inertial fluctuations than the amplitude variations. So the semidiurnal residues are basically associated with phase variations of the semi-diurnal tidal waves.

The relationship between sub-inertial fluctuations in the flow and semi-diurnal residues generation can be explained in terms of the non- linear interaction between tidal and sub-inertial flows, occuring mainly through the advective term in the momentum balance equation.

\section{ACKNOWLEDGEMENTS}

The authors would like to thank Dr. García Lafuente and an anonymous reviewer for helpful comments on the manuscript.

\section{REFERENCES}

Amin, M. - 1976. The fine resolution of tidal harmonics. Geophy. J. R. Astro. Soc., 44: 293-310.
Armi, L. And D. Farmer. - 1988. The Flow of Mediterranean Water Through the Strait of Gibraltar. Prog. Oceanog., 21: 1-105.

Box, G.E.P. and G.M. Jenkins. - 1970. Time series analysis, forecasting and control. Holden day, Inc., San Francisco.

Bray, N.A., C.D. Winant, T.H. Kinder and J. Candela. - 1990. Generation and kinematics of the internal tide in the Strait of Gibraltar. In: L.J. Pratt, (ed.), The Physical Oceanography of Sea Straits pp. 477-491. Kluwer Acad. Publ.

Bruno, M., R. Mañanes, J.J. Alonso and L. Tejedor. - 1996a. Meteorological tides and their relationship with tidal semi-diurnal residues. Sci. Mar, 60(4): 451-459.

Candela, J., C.D. Winant and A. Ruíz. - 1990. Tides in the strait of Gibraltar. J. Geophys. Res., 95: 7317-7335.

Candela, J., C.D. Winant and H.L. Bryden. - 1989. Meteorologically forced sub-inertial flows trough the Strait of Gibraltar. $J$. Geophys. Res., 94: 12667-12674.

Farmer, D. and L. Armi. - 1988. The Flow of Atlantic Water Through the Strait of Gibraltar. Prog. Oceanog., 21: 1-105.

Foreman, M.G.G. - 1976. Manual for tidal heights and prediction. Pacific Marine Sciences report 77-10 Inst. of Ocean sciences, Patrice bay.

García, J.M.. - 1986. Variabilidad del nivel del mar en el Estrecho de Gibraltar: Marea y oscilaciones residuales. Ph. D. Thesis, Universidad de Málaga.

Garrett,C., J. Akerly and K. Thompson. - 1989. Low frequency fluctuations in the Strait of Gibraltar from MEDALPEX sea level data. J.Phys.Oceangr., 19: 1682-1696.

Godin, G. - 1972. The analysis of tides. University of Toronto, Toronto.

Mañanes, R. - 1996. Interacción no lineal entre los flujos subinerciales y mareales asociados al régimen barotrópico en el Estrecho de Gibraltar. Ph. D. thesis, Universidad de Cádiz.

Mañanes, R., M. Bruno, J. Alonso, B. Fraguela and L. Tejedor. 1998. The nonlinear interaction between tidal and sub-inertial flows in the Strait of Gibraltar. Oceanol. Acta 21: 33-46,

Munk, W.H., B. Zetler and G.W. Groves. - 1964. Tidal cusps. Geophy. J. R. Astro. Soc., 10: 211-219.

Pillbsbury, R.D., D. Barstow., J.S. Botero., C. Millero., B. More., G. Pittock., D.C. Root., J. simpkims III., R.E. Still and H.L. Bryden . - 1987. Gibraltar Experiment: Current measurements in the Strait of Gibraltar. Oregon State University Technical report., 87: 29-284.

Rossiter J.R. and G.W. Lennon. - 1968. An intensive analysis of shallow water tides. Geophys. J. R. astr. Soc., 16: 275-293.

Tejedor, L., A. Izquierdo., B.A. Kagan, and D.V. Sein. (in press). Simulation of the semi-diurnal tides in the Strait of Gibraltar. $J$. Geophys. Res.

Scient. ed.: J. Font 